\title{
Dissociative electron attachment studies with hyperthermal Rydberg atoms
}

\author{
S. Buathong and F. B. Dunning \\ Department of Physics and Astronomy, Rice University, Houston, Texas 77005-1892, USA
}

(Received 23 July 2018; accepted 29 August 2018; published online 11 September 2018)

\begin{abstract}
Earlier studies of the velocity distributions of heavy-Rydberg ion-pair states formed in collisions between potassium Rydberg atoms with low-to-intermediate values of $n, 10 \lesssim n \lesssim 15$, and targets that attach free low-energy electrons have shown that such measurements can provide a window into the dynamics of dissociative electron capture. Here we propose that the reaction dynamics can be explored in much greater detail through studies using hyperthermal Rydberg atoms. This is demonstrated using, as an example, helium Rydberg atoms and a semi-classical Monte Carlo collision code developed specifically to model the dynamics of Rydberg electron transfer in collisions between Rydberg atoms and attaching targets. The simulations show that the outcome of collisions is sensitive not only to the lifetime and decay energetics of the excited intermediate negative ion formed upon initial Rydberg electron capture but also to the radial electron probability density distribution in the Rydberg atom itself, i.e., to its $\ell$ value. Published by AIP Publishing. https://doi.org/10.1063/1.5049597
\end{abstract}

\section{INTRODUCTION}

Atoms in Rydberg states with large values of principal quantum number $n$ possess physical characteristics quite unlike those normally associated with atoms in ground or lowlying states. In particular, since the Bohr radius of an atom scales as $n^{2}$, they are physically very large. For sufficiently large values of $n, n \gtrsim 10$, the separation between the Rydberg electron and core ion becomes larger than the ranges typically associated with ion-molecule and electron-molecule interactions. Thus in collisions with neutral targets, Rydberg atoms behave not as an atom but rather as a pair of independent scatterers. Many collision processes are dominated by the (binary) Rydberg electron-target interaction, including collisions with targets that attach free low-energy electrons where capture of the Rydberg electron results in the formation of a positivenegative ion pair. For large values of $n$, the size of the Rydberg electron cloud is such that the separation between the product ions is sufficient that their mutual post-attachment electrostatic interactions are weak and, even in thermal-energy collisions, the great majority are able to separate as free ions. However, as $n$, and the size of the electron cloud, decreases, the initial separations between the product ion pairs also decrease and an increasing fraction of them become electrostatically bound, leading to the formation of molecular ion-pair states in which the ions orbit each other at relatively large separations weakly bound by their mutual Coulomb attraction. For targets that undergo dissociative electron capture, this results in reactions of the type

$$
\mathrm{K}(n \mathrm{p})+\mathrm{ABC} \rightarrow \mathrm{K}^{+}+\mathrm{ABC}^{-*} \rightarrow\left\{\begin{array}{l}
\mathrm{K}^{+} \cdots \mathrm{C}^{-}+\mathrm{AB} \\
\mathrm{K}^{+} \cdots \mathrm{BC}^{-}+\mathrm{A},
\end{array}\right.
$$

where $\mathrm{ABC}^{-*}$ denotes the excited intermediate that is formed upon initial electron capture and that dissociates to produce $\mathrm{C}^{-}$ or $\mathrm{BC}^{-}$charged fragments, and $\mathrm{K}^{+} \ldots \mathrm{C}^{-}$and $\mathrm{K}^{+} \ldots \mathrm{BC}^{-}$represent the bound ion pairs. Since many of the properties of such ion-pair states parallel those of Rydberg atoms, they are also frequently termed heavy-Rydberg states. ${ }^{1}$ Such states have been generated not only through Rydberg atom collisions ${ }^{2-6}$ but also by direct photoexcitation of ground-state diatomic molecules. ${ }^{1,7-10}$

Earlier Rydberg atom studies undertaken using a target gas cell and $\mathrm{K}(n \mathrm{p})$ Rydberg atoms with low-to-intermediate values of $n, 10 \lesssim n \lesssim 15$, excited in a tightly collimated thermal potassium atom beam have shown that measurements of the velocity and angular distributions of the product ion-pair states can provide a window into the properties of the excited intermediates formed upon initial electron capture, including their lifetimes and the energetics of their decay. The information that could be extracted from these earlier Rydberg measurements, however, was limited by experimental factors including the use of thermal potassium Rydberg atoms and a gas cell to contain the target gas. Here we propose that much greater detail regarding the reaction dynamics can be obtained from Rydberg collision experiments by more precisely controlling the initial collision conditions using a crossed target beam and by exploiting supersonic beam sources ${ }^{11-13}$ which permit generation of hyperthermal helium Rydberg atom beams that have a narrow velocity distribution. Helium Rydberg atom velocities of $\sim 2 \times$ $10^{3} \mathrm{~m} \mathrm{~s}^{-1}$ have been achieved using a supersonic nozzle beam source operating with a room-temperature pulsed valve, but these velocities might be increased to values approaching $3 \times$ $10^{3} \mathrm{~m} \mathrm{~s}^{-1}$ by the use of a valve that can operate at high temperatures. The highest helium atom beam velocities achieved using a heated supersonic nozzle beam source are $\sim 4 \times 10^{3} \mathrm{~m} \mathrm{~s}^{-1}$. ${ }^{14}$ Here we explore the new capabilities that hyperthermal Rydberg atom sources provide for the study of dissociative electron capture using a semi-classical Monte Carlo collision code developed specifically to probe the dynamics of Rydberg 
electron transfer reactions and show, using a selection of target species, that the predicted collisional outcomes are particularly sensitive to the assumed lifetime (and decay energetics) of the excited intermediate. In consequence, comparisons between theory and experiment promise improved values for these parameters and a deeper insight into electron capture processes in general. Furthermore, we show that the outcome of collisions can become sensitive to the radial electron probability density distribution in the Rydberg atom itself, i.e., to its $\ell$-value.

\section{COLLISION MODEL}

The velocity and angular distributions of the product ionpair states that result from Rydberg collisions are simulated using a semi-classical Monte Carlo collision code that models the detailed kinematics of electron transfer reactions. ${ }^{2-6,15}$ The model is based on the independent particle picture, and attachment is viewed as resulting from a binary interaction between the Rydberg electron and target particle. The initial velocities of the Rydberg atom and target particle are selected at random from the appropriate distribution of these quantities. The probability of electron capture at some point is taken to be proportional to the local electron probability density which is computed using hydrogenic wavefunctions and is averaged over the possible values of $m_{\ell}$. (For low- $\ell$ states with sizable quantum defects, $\delta$, hydrogenic states with the same value of $\ell$ but the value of $n$ closest to the effective principal quantum number $n^{*}=n-\delta$ are used.) Following capture, the motions of the core ion and the excited intermediate negative ion are computed using classical mechanics. After some time interval, governed by the assumed lifetime, $\tau$, of the intermediate, dissociation is presumed to occur. If dissociation is immediate, i.e., if the electron is captured directly into an antibonding orbital, it is reasonable to expect that the great majority of the excess energy of reaction, $E_{E}$, will appear in translation. By contrast, if the intermediate is longer lived, i.e., if the lifetime is in the order of at least a few vibrational periods, some redistribution of the excess energy within the intermediate might occur prior to dissociation when part of the excess energy can appear as rovibrational excitation of the fragments rather than in translation. In the limit that the lifetime of the intermediate is sufficient that the excess energy can be statistically redistributed within the intermediate, unimolecular decay theory predicts a Boltzmann-like translational energy release distribution of the form $e^{-\epsilon / \bar{\epsilon}}$, where $\bar{\epsilon}$ is the mean translational energy release. ${ }^{16}$ Upon dissociation, the angular distribution of the fragments is taken to be isotropic in the rest frame of the intermediate. The negative ion velocity in the laboratory frame is then computed to determine the kinetic energy of relative motion of the ion pair and hence their total (kinetic plus potential) energy and whether or not they are bound. If bound, the trajectory of the resulting ion pair is calculated.

In earlier experimental studies of the velocity and angular distributions of ion-pair states produced in Rydberg atom collisions, only those ion pairs that travel within a few degrees of a fixed (horizontal) plane, i.e., atom pairs that have only a small component of transverse (vertical) velocity, were measured. ${ }^{2-6}$
Such "two-dimensional" velocity distributions reflect more directly the reaction dynamics and are more straight forward to interpret. Such ion pairs are therefore identified in the simulations and it is their velocity and angular distributions that are built up by considering the outcome of many collision events. Calculations using different assumed input parameters, i.e., different intermediate lifetimes (and decay energetics), are then used to assess how sensitive the predicted distributions are to these parameters and hence the level of detailed information regarding them that might be extracted from comparisons between theory and experiment.

To demonstrate the gains that can be achieved using hyperthermal helium Rydberg atoms, the predicted velocity distributions for ion-pair states produced in $\mathrm{K}(12 \mathrm{p})$ collisions with target gases contained in a gas cell (conditions which mirror those of earlier experimental studies) are compared to those expected when using faster velocity-selected helium Rydberg atoms and a crossed target beam. Although results are only included for a few select target species, calculations indicate that similar gains can be achieved with a broad range of targets.

\section{RESULTS AND DISCUSSION}

Consider initially dissociative Rydberg electron transfer to $\mathrm{CCl}_{4}$ which can lead to ion-pair formation via the reaction

$$
\mathrm{X}^{* *}+\mathrm{CCl}_{4} \rightarrow \mathrm{X}^{+}+\mathrm{CCl}_{4}^{-*} \rightarrow \mathrm{X}^{+} \cdots \mathrm{Cl}^{-}+\mathrm{CCl}_{3} \text {. }
$$

Earlier studies of the velocity distribution of free $\mathrm{K}^{+}$ions formed in $\mathrm{K}(55 \mathrm{p})-\mathrm{CCl}_{4}$ collisions (where post-attachment electrostatic interactions are negligible) have shown that the lifetimes of the $\mathrm{CCl}_{4}^{-*}$ intermediates are sufficient to allow near-statistical redistribution of the excess energy of reaction into internal motions prior to dissociation resulting in a Boltzmann-like translational energy release distribution of the form $\mathrm{e}^{\varepsilon / \bar{\varepsilon}}$ with $\bar{\varepsilon}=0.1 \mathrm{eV} .^{17}$ The lifetime of the intermediate has been explored through measurements of the angular distribution of free ions and of bound ion pairs produced in low- $n$ collisions using K(12p) (and K(14p)) Rydberg atoms. The studies of ion-pair formation were conducted in a pulsed mode. ${ }^{2,3}$ Short laser pulses were used to excite Rydberg atoms in the presence of target gas in a small volume located near the center of a gas cell. The effective lifetimes of the Rydberg atoms were short, $\sim 1-2 \mu \mathrm{s}$, and they decayed before moving any significant distance from their points of formation. As a result, collisions lead to the production of spatially and temporally localized groups of ion-pair states. The velocity and angular distributions of those ion-pair states that travel close to the horizontal plane were determined by measuring their spatial distributions as a function of the time delay, $t_{D}$, following their creation. The ion-pair states were detected by electric-field-induced dissociation. The critical field, $F_{c}$, at which dissociation occurs is related to the binding energy by the approximate expression ${ }^{18}$

$$
E_{b}(\mathrm{meV})=14\left[F_{c}\left(\mathrm{kV} \mathrm{cm}^{-1}\right)\right]^{1 / 2} .
$$

In initial experiments, practical considerations limited the size of the electric fields that could be applied experimentally to $\lesssim 7.5 \mathrm{kV} \mathrm{cm}^{-1}$ and only states with values of $E_{b} \lesssim 30-40 \mathrm{meV}$ 
could be detected. However, in principle, it should be possible to apply fields of up to $\sim 15$ to $\sim 20 \mathrm{kV} \mathrm{cm}^{-1}$ permitting detection of ion-pair states with binding energies of up to $\sim 60 \mathrm{meV}$. In the following, we limit discussion of ion-pair velocity distributions to those for ion-pair states that travel close to the horizontal plane and have binding energies $E_{b} \lesssim 50 \mathrm{meV}$.

Figure 1(a) shows simulated spatial distributions for $\mathrm{K}^{+} \ldots \mathrm{Cl}^{-}$ion pairs formed in $\mathrm{K}(12 \mathrm{p})-\mathrm{CCl}_{4}$ collisions. These calculations assume that $\mathrm{K}(12 \mathrm{p})$ atoms have a thermal $(600 \mathrm{~K})$ velocity distribution and that the $\mathrm{CCl}_{4}$ target gas is contained in a gas cell. In accordance with earlier measurements, the calculations also assume that dissociation results in a Boltzmannlike translational energy release distribution with $\bar{\varepsilon}=0.1 \mathrm{eV}$. Results are presented for several different assumed mean intermediate lifetimes $\tau$, all sufficient to allow near-statistical redistribution of the excess energy. A time delay $t_{D}=30 \mu \mathrm{s}$ is selected. Inspection of Fig. 1 shows that, whereas the predicted distributions do depend on the choice of $\tau$, the dependence is rather weak. (Earlier measurements of free-ion production point to a lifetime $\tau \sim 8$ ps. ${ }^{17}$ ) This lack of sensitivity limits the amount of detailed information regarding the lifetime that can be extracted from comparisons between theory and experiment.
Figure 1(b) shows the results of simulations which assume that, as a first step toward better defining the initial collision conditions, the gas cell is replaced by a crossed (thermal) molecular beam. As might be expected, the use of a crossed target beam leads to marked left-right asymmetries in the predicted angular distributions. However, the predicted spatial distributions remain insensitive to the choice of intermediate lifetime.

The improvements in sensitivity that can be achieved by the use of hyperthermal helium Rydberg atoms (coupled with a crossed target beam) are illustrated in Fig. 1(c) which shows calculated spatial distributions for $\mathrm{He}^{+} \ldots \mathrm{Cl}^{-}$ ion pairs formed in collisions involving hyperthermal $(v=2$ $\left.\times 10^{3} \mathrm{~m} \mathrm{~s}^{-1}\right) \mathrm{He}(10 \mathrm{p})$ atoms. Comparison with the results for $\mathrm{K}(12 \mathrm{p})$ atoms shows that the predicted distributions possess more structure and are much more sensitive to the choice of the intermediate lifetime $\tau$. Thus comparisons between theory and experiment promise a much improved value for this parameter.

As a further demonstration of the advantages afforded by studying collisions involving hyperthermal Rydberg atoms, consider ion-pair formation through dissociative electron transfer to $\mathrm{BrCN}$ which proceeds via the a)

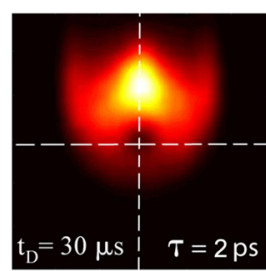

b)

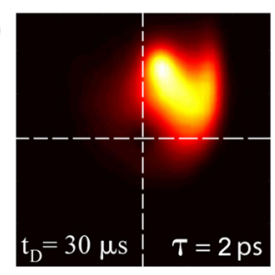

c) $\mathrm{n}=10$

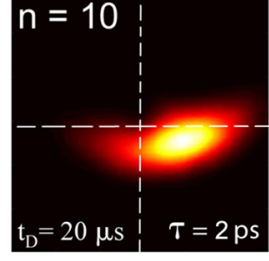

d)

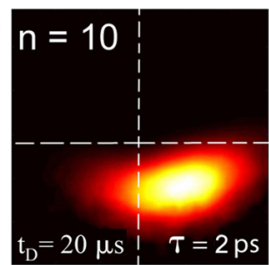

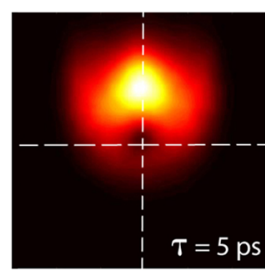
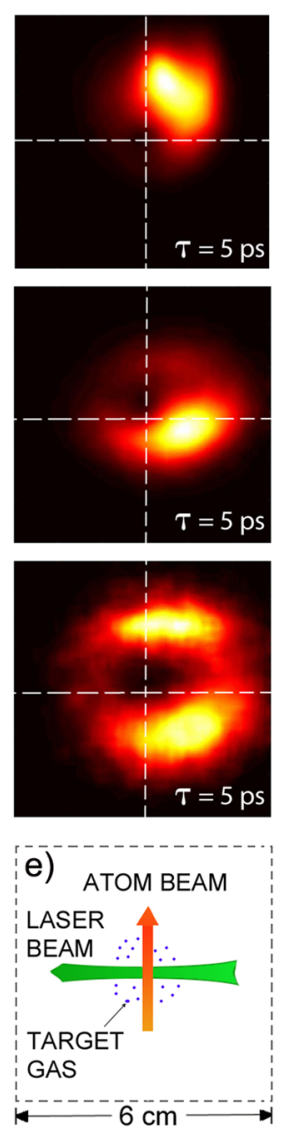
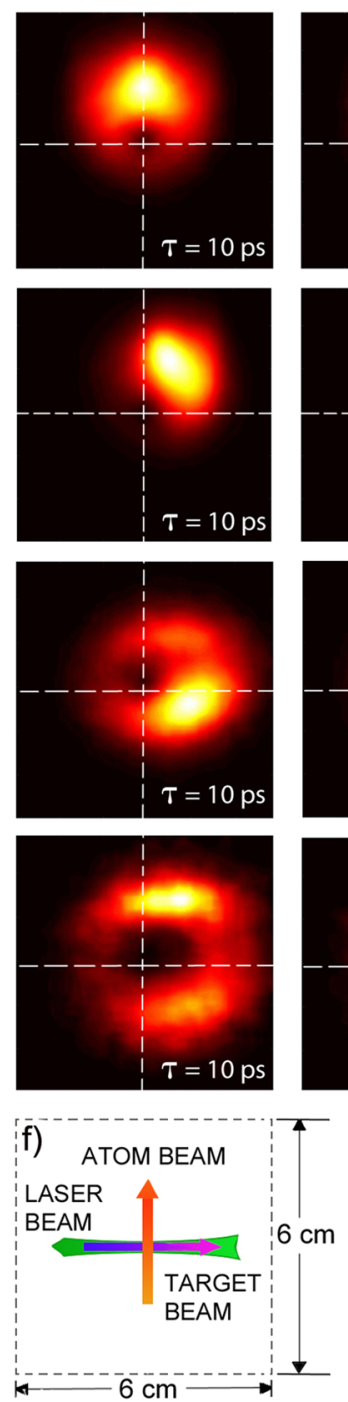
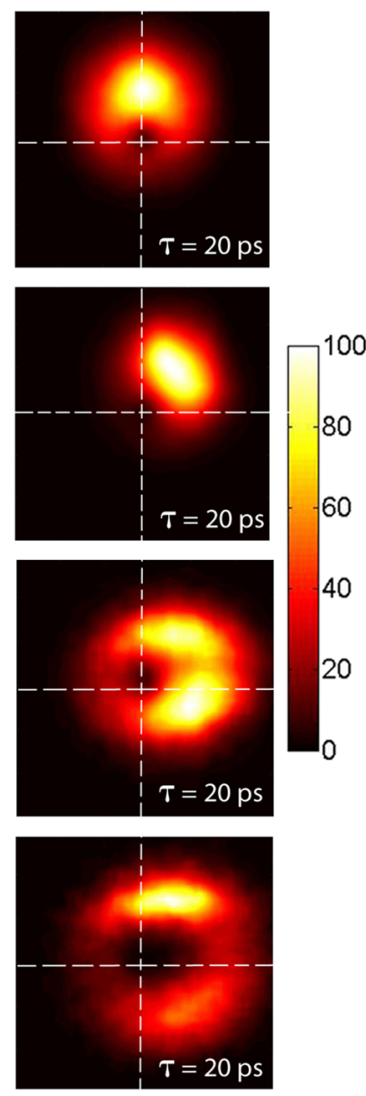

FIG. 1. Calculated spatial distributions for $\mathrm{K}^{+} \ldots \mathrm{Cl}^{-}$and $\mathrm{He}^{+} \ldots \mathrm{Cl}^{-}$ion pairs with binding energies $\lesssim 30 \mathrm{meV}$ formed in (a) and (b) $\mathrm{K}(12 \mathrm{p})-\mathrm{CCl}_{4}$, (c) $\mathrm{He}(10 \mathrm{p})-\mathrm{CCl}_{4}$, and (d) $\mathrm{He}(n=10$, $\ell=9)-\mathrm{CCl}_{4}$ collisions for the values of the mean intermediate lifetimes $\tau$ indicated. The simulations assume a mean translational energy release $\bar{\varepsilon}=0.1 \mathrm{eV}$ and consider only ion pairs formed traveling close to the $x y$ plane (see the text). The spatial distributions are for a target gas contained (a) in a gas cell and (b)(d) in a crossed molecular beam. The calculations assume the time delays $t_{D}$ shown, a $600 \mathrm{~K}$ thermal velocity distribution for the $\mathrm{K}(12 \mathrm{p})$ atoms, and a $\mathrm{He}(10 \mathrm{p})$ velocity of $2 \times 10^{3} \mathrm{~m} \mathrm{~s}^{-1}$. The distributions in each panel are normalized to the peak value in that panel. (e) and (f) show the experimental arrangements assumed in the calculations. The ion pairs are initially formed at the center of each panel. 
reaction

$$
\mathrm{X}^{* *}+\mathrm{BrCN} \rightarrow \mathrm{X}^{+}+\mathrm{BrCN}^{-*} \rightarrow \mathrm{X}^{+} \cdots \mathrm{CN}^{-}+\mathrm{Br}
$$

Previous work using K(55p) Rydberg atoms has shown that the translational energy release distribution that accompanies dissociation can be approximated by a Gaussian function with a full width at half maximum of $\sim 0.05 \mathrm{eV}$ centered on $\bar{\epsilon} \sim 0.1 \mathrm{eV} .{ }^{19}$ The latter value is somewhat less than the available excess energy of reaction, $E_{E}$, estimated to be $\sim 0.14 \mathrm{eV}$, indicating that some redistribution of this energy into the $\mathrm{CN}^{-}$ fragment must have occurred. However, the energy deficit, $\sim 0.04 \mathrm{eV}$, is much less than the calculated vibrational spacing for $\mathrm{CN}^{-}$ions $(\sim 0.25 \mathrm{eV})$ whereupon dissociation must lead to the formation of $\mathrm{CN}^{-}$ions in the ground vibrational state. As a consequence, any energy deficit must appear as rotational excitation of the $\mathrm{CN}^{-}$fragment. Since the rotational constant for $\mathrm{CN}^{-}$is small, $B\left(\mathrm{CN}^{-}\right) \sim 0.23 \mathrm{meV},{ }^{20}$ an energy deficit of $0.04 \mathrm{eV}$ would suggest the formation of $\mathrm{CN}^{-}$ions with rotational quantum numbers $J \sim 13$, larger than those characteristic of a room-temperature thermal distribution.

Information on the rotational distribution can be obtained from measurements at lower $n$ by comparing the results to simulations which assume different product $\mathrm{CN}^{-}$rotational energy distributions and then subtracting these distributions from the excess energy of reaction to obtain the corresponding translational energy release distributions. Figure 2(a) shows simulated spatial distributions for $\mathrm{K}^{+} \ldots \mathrm{CN}^{-}$ion pairs formed in $\mathrm{K}(12 \mathrm{p})-\mathrm{BrCN}$ collisions using a crossed target beam. An intermediate $\mathrm{BrCN}^{-*}$ lifetime of $20 \mathrm{ps}$ is assumed consistent with earlier measurements. ${ }^{19}$ Inspection of Fig. 2(a) shows that the predicted distributions are not strongly dependent on the choice of rotational distribution, i.e., temperature. By contrast, as seen in Fig. 2(b), the predicted spatial distributions for $\mathrm{He}^{+} . . \mathrm{CN}^{-}$ion pairs formed through $\mathrm{He}(10 \mathrm{p})-\mathrm{BrCN}$ collisions when using a hyperthermal $\mathrm{He}(10 \mathrm{p})$ atom beam are rather sensitive to the assumed rotational distribution of the $\mathrm{CN}^{-}$ions. Indeed, the sensitivity appears such that it should be possible, through calculations using non-thermal trial distributions, to get a relatively detailed measure of the actual rotational energy distribution.
Figure 1 also includes arrival position distributions calculated for $\mathrm{He}^{+} \ldots \mathrm{Cl}^{-}$ion-pair states formed in $\operatorname{He}(n=10$, $\ell=9)-\mathrm{CCl}_{4}$ collisions. The spatial distributions are rather different from those predicted for the 10p state but are again sensitive to the choice of intermediate lifetime. The differences in the predicted spatial distributions result from differences in the Rydberg electron radial probability density distributions. These distributions govern the radial separations at which Rydberg electron capture occurs and hence the strength of the subsequent post-attachment electrostatic interactions.

An attractive target with which to evaluate $\ell$-dependences in Rydberg atom collisions is $\mathrm{CF}_{3} \mathrm{I}$ for which ion-pair formation can occur through reactions of the type

$$
\mathrm{X}^{* *}+\mathrm{CF}_{3} \mathrm{I} \rightarrow \mathrm{X}^{+}+\mathrm{CF}_{3} \mathrm{I}^{-*} \rightarrow \mathrm{X}^{+} \cdots \mathrm{I}^{-}+\mathrm{CF}_{3}
$$

Earlier work ${ }^{3,21,22}$ has shown that, following electron capture, essentially all the excess energy of reaction, $E_{E} \sim 0.8 \mathrm{eV}$, appears in translation pointing to immediate dissociation before any redistribution of the excess energy to internal motions can occur, behavior that is consistent with direct capture into an antibonding orbital. The resulting $\mathrm{I}^{-}$velocity (in the molecule-fixed frame), $\sim 7 \times 10^{2} \mathrm{~m} \mathrm{~s}^{-1}$, is much larger than the initial thermal velocity of the target molecule. In consequence, the initial velocity distribution of the $\mathrm{I}^{-}$ ions in the laboratory-fixed frame is narrow. Furthermore, the very short $\mathrm{CF}_{3} \mathrm{I}^{-*}$ intermediate lifetime does not allow time for significant scattering of the $\mathrm{He}^{+}$Rydberg core ion prior to dissociation. The initial conditions immediately post-dissociation are thus particularly well defined allowing more detailed analysis and interpretation of experimental data.

Figure 3 shows calculated spatial distributions for $\mathrm{He}^{+} \cdots \mathrm{I}^{-}$ion pairs with binding energies $30 \lesssim E_{B} \lesssim 50 \mathrm{meV}$ formed in $\mathrm{He}(11 \ell)-\mathrm{CF}_{3} \mathrm{I}$ collisions for several different values of $\ell$. [Such ion pairs can be identified, with the aid of Eq. (3), by applying a ramped electric field and measuring the time, i.e., field, at which dissociation occurs.] Although the spatial distributions for low- $\ell, \ell \leq 4$, states display marked structure, this structure is not particularly sensitive to the choice of $\ell$. For larger $\ell$ values, however, the calculated spatial distributions become more strongly dependent on $\ell$. a)

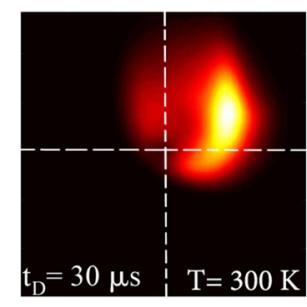

b)

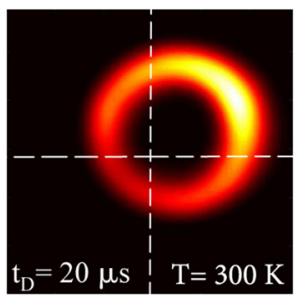

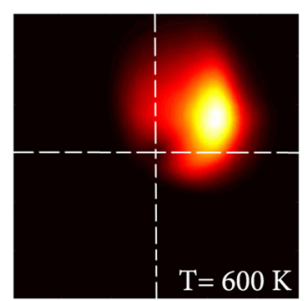

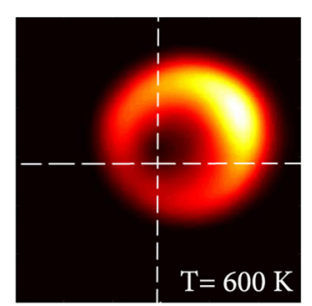

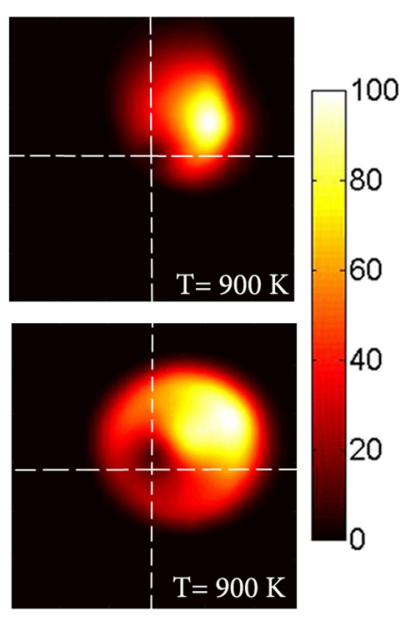

FIG. 2. Calculated spatial distributions for $\mathrm{K}^{+} \ldots \mathrm{CN}^{-}$and $\mathrm{He}^{+} \ldots \mathrm{CN}^{-}$ion pairs with binding energies $\lesssim 30 \mathrm{meV}$ formed in (a) $\mathrm{K}(12 \mathrm{p})-\mathrm{BrCN}$ and (b) $\mathrm{He}(10 \mathrm{p})-\mathrm{BrCN}$ collisions. The simulations assume different $\mathrm{CN}^{-}$rotational distributions characterized by the temperatures, $T$, indicated (see the text), a $\mathrm{BrCN}^{-*}$ intermediate lifetime $\tau=20 \mathrm{ps}$, a $600 \mathrm{~K}$ thermal velocity distribution for the $\mathrm{K}(12 \mathrm{p})$ atoms, a $\mathrm{He}(10 \mathrm{p})$ velocity of $2.5 \times 10^{3} \mathrm{~m} \mathrm{~s}^{-1}$, the time delays $t_{D}$ indicated, and the use of a crossed target beam. 
a)
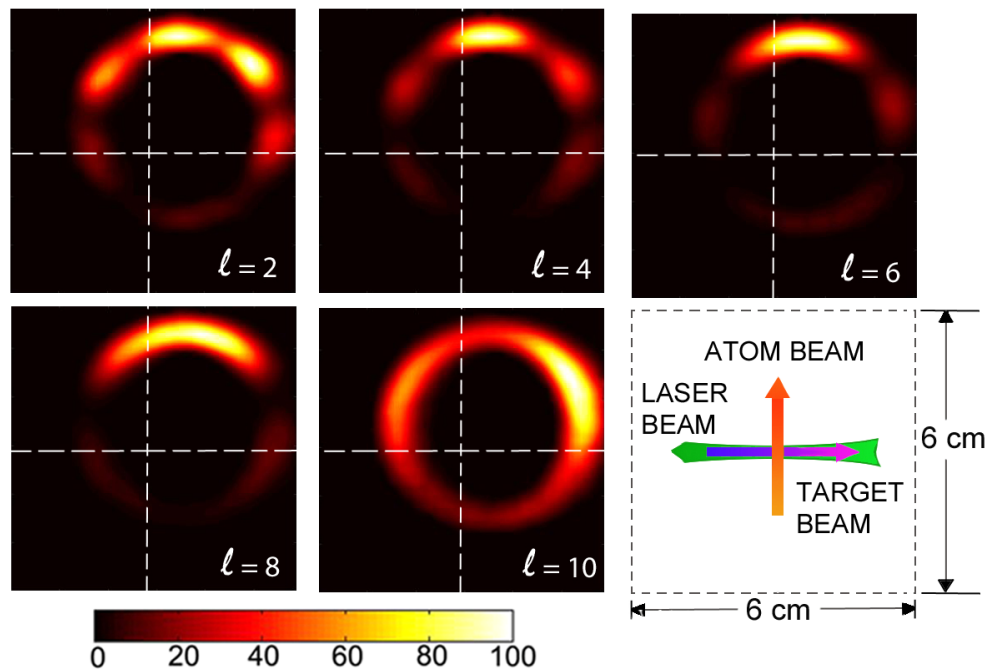

b)

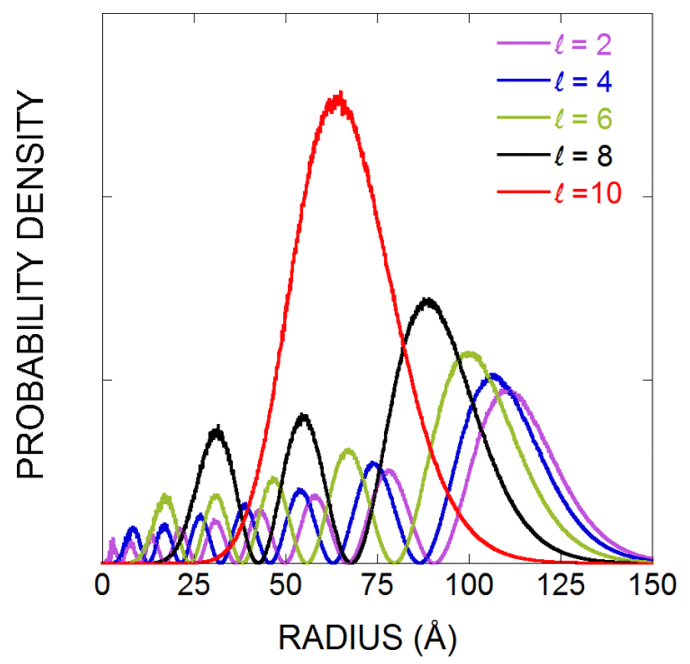

FIG. 3. (a) Calculated spatial distributions of $\mathrm{He}^{+} \ldots \mathrm{I}^{-}$ion pairs with binding energies $30 \lesssim E_{B} \lesssim 50 \mathrm{meV}$ formed traveling near the $x y$ plane in $\mathrm{He}(11 \ell)-\mathrm{CF}_{3} \mathrm{I}$ collisions for the values of $\ell$ indicated. The velocity of the $\mathrm{He}(11 \ell)$ atoms is $3 \times 10^{3} \mathrm{~m} \mathrm{~s}^{-1}$. The calculations assume a Gaussian translational energy release distribution of $50 \mathrm{meV}$ FWHM centered on $\epsilon=0.8 \mathrm{eV}$ (see the text), an intermediate $\mathrm{CF}_{3} \mathrm{I}^{-*}$ lifetime of $\tau=0 \mathrm{ps}$, and a time delay $t_{D}=30 \mu \mathrm{s}$. (b) Radial electron probability density distributions for $\mathrm{He}(n=11)$ Rydberg states with the values of $\ell$ indicated.
The structure and $\ell$-dependences seen in Fig. 3 can be understood with reference to Fig. 4 which shows the arrival position distributions to be expected if capture is restricted to the different narrow radial ranges defined by the peaks $P_{1}$ to $P_{10}$ in Fig. 4(b). To emphasize the relative importance of capture over the different radial intervals, the spatial distributions in Fig. 4(a) are displayed using a common color bar and each corresponds to the same initial number of collision events.

The general features of the position distributions seen in Fig. 4 can be readily understood. If capture occurs at large radii $(R \gtrsim 90 \AA)$, the initial electrostatic binding of the ion pair is small and only ion pairs with small initial kinetic energies of relative motion, $E_{K r e l}$, will remain bound which requires that the $\mathrm{I}^{-}$ion be formed traveling in the same general direction as the Rydberg core ion, i.e., close to the forward direction. The associated ion-pair spatial distribution is therefore also strongly peaked near the forward direction. The ion-pair velocities, $\sim 8 \times 10^{2} \mathrm{~m} \mathrm{~s}^{-1}$, are governed by momentum conservation (and the large mass of the $\mathrm{I}^{-}$ion). As the separation at which capture occurs decreases, the initial electrostatic binding increases to the point that few ion-pair states formed traveling near the forward direction have binding energies in the range of interest. The initial relative kinetic energy, $E_{\text {Krel }}$, however, is increased if the $\mathrm{I}^{-}$ ions are initially formed with substantial transverse components of velocity. Formation of weakly bound ion pairs again becomes possible which, because of momentum conservation, travel at sizable "scattering" angles, $\theta$, to the forward direction. As capture occurs at ever smaller radii, the increases in initial electrostatic binding must be offset by increases in $E_{\text {Krel }}$ which requires that the initial velocity of the $\mathrm{I}^{-}$ion progressively rotate so as to become increasingly "antiparallel" to that of the $\mathrm{He}^{+}$ion. The maximum value of $E_{\text {Krel }}$ is achieved when the $\mathrm{He}^{+}$and $\mathrm{I}^{-}$ions are formed traveling in opposite directions but is insufficient to allow significant creation of weakly bound ion pairs if capture occurs at separations $\$ 50 \AA$.

The spatial distributions seen in Fig. 4 enable a detailed analysis of the predicted distributions for the different $\mathrm{He}(11 \ell)$ states shown in Fig. 3. The radial electron probability density distribution for $\ell=2$ states has multiple peaks including the ones centered at $\sim 58,78$, and $110 \AA$. The spatial distribution associated with ion-pair formation is therefore expected to be a superposition of those associated with these different features. (Capture involving the features at smaller radii does not contribute to the production of weakly bound ion pairs.) Inspection of Fig. 4 shows that capture at radial separations corresponding to the outermost peak in the radial 
a)
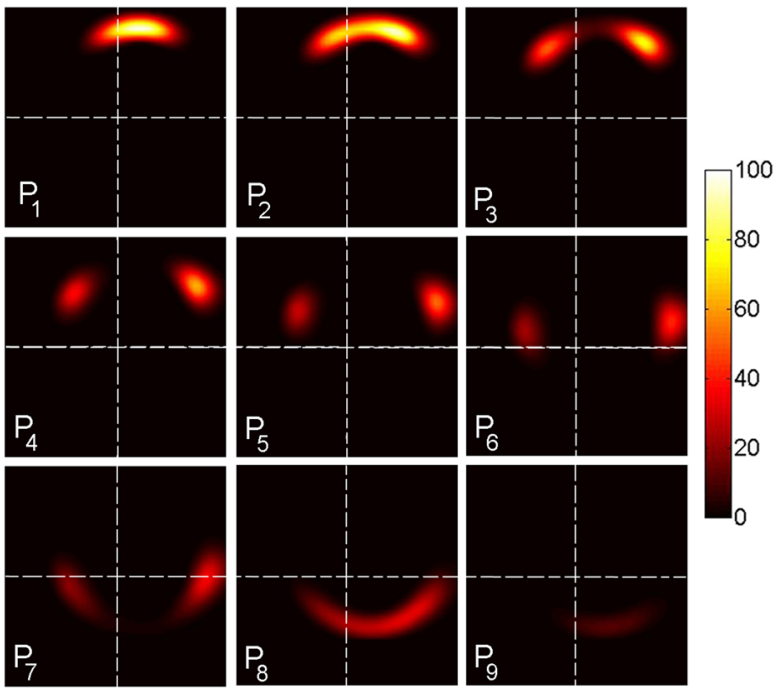

b)

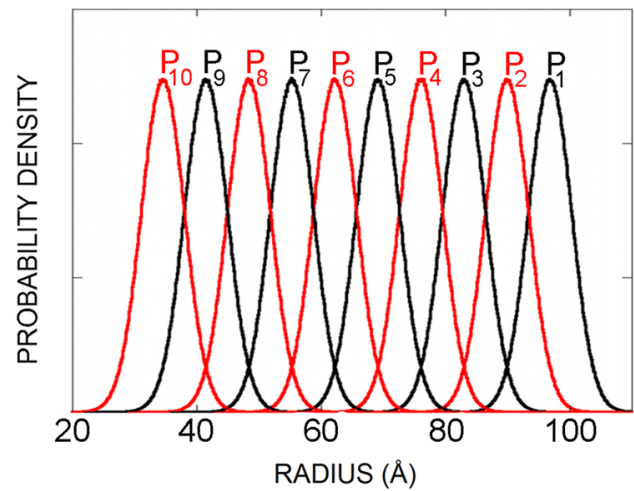

FIG. 4. (a) Calculated spatial distributions for $\mathrm{He}^{+} \cdots \mathrm{I}^{-}$ion pairs with binding energies $30 \lesssim E_{B} \lesssim 50 \mathrm{meV}$ formed traveling near the $x y$ plane if capture is restricted to the narrow ranges of internuclear separations defined by the peaks $P_{1}$ to $P_{10}$ shown in (b). The spatial distributions are all displayed using a common color bar (see the text) and a helium velocity of $3 \times 10^{3} \mathrm{~m} \mathrm{~s}^{-1}$ is assumed.

distribution accounts for the strong feature seen near the forward direction in the predicted spatial distribution. Capture at smaller separations corresponding to the peak at $\sim 78 \AA$ gives rise to the maxima seen at scattering angles of $\sim \pm 45^{\circ}$, while capture in the neighboring peak at $\sim 58 \AA$ accounts for the small features seen at scattering angles of $\sim \pm 90^{\circ}$. The radial distribution for $\ell=4$ states again has three peaks in the radial interval of interest. Whereas each occurs at a somewhat smaller radius than for the $\ell=2$ states, the predicted spatial distribution is nonetheless similar to that for $\ell=2$ states. For $\ell=6$ states, the radial distribution contains only two peaks in the range of interest located at $\sim 68$ and $\sim 100 \AA$ A. As a result, the predicted spatial distribution contains less structure. The radial peak at $\sim 100 \AA$ accounts for the strong peak seen near the forward direction, the peak at $\sim 68 \AA$ the smaller features at scattering angles of $\sim \pm 70^{\circ}$. The major contribution to ion-pair production for $\ell=8$ states is produced by the radial peak at $\sim 90 \AA$ with only a minor contribution originating from the peak at $\sim 55 \AA$. In consequence, the corresponding spatial distribution contains only a single feature that is centered close to the forward direction. The radial distribution for $\ell=10$ states contains a single broad peak with a maximum at a radius of $\sim 65 \AA$. This gives rise to a broad spatial distribution with maxima at scattering angles of $\sim \pm 60^{\circ}$.

As demonstrated by the above examples, measurements of the velocity distribution of ion-pair states formed in collisions with hyperthermal Rydberg atoms are particularly sensitive not only to the lifetime of the intermediates and but also to the angular momentum state of the Rydberg atoms themselves. Furthermore, while helium Rydberg atoms were selected for this study because the production of hyperthermal helium Rydberg atoms has been demonstrated (together with the control of their velocities ${ }^{13}$ ), simulations suggest that similar improvements in sensitivity are to be expected when using other light Rydberg species such as hydrogen or lithium. The present work shows that, although few measurements of collisions involving hyperthermal Rydberg atoms have been undertaken, ${ }^{23,24}$ they promise to open up interesting new avenues for the study of reaction dynamics as well as the characteristics of the Rydberg atoms themselves.

\section{ACKNOWLEDGMENTS}

It is a pleasure to acknowledge valuable discussions with S. D. Hogan. This research was supported by the Robert A. Welch Foundation under Grant No. C-0734.

${ }^{1}$ E. Reinhold and W. Ubachs, Mol. Phys. 103, 1329 (2005).

${ }^{2}$ C. H. Wang, M. Kelley, S. Buathong, and F. B. Dunning, J. Chem. Phys. 140, 234306 (2014).

${ }^{3}$ S. Buathong, M. Kelley, C. H. Wang, and F. B. Dunning, Chem. Phys. Lett. 618, 153 (2015).

${ }^{4}$ M. Kelley, S. Buathong, and F. B. Dunning, J. Phys.: Conf. Ser. 635, 012024 (2015).

${ }^{5}$ S. Buathong, M. Kelley, and F. B. Dunning, J. Chem. Phys. 145, 134309 (2016).

${ }^{6}$ M. Kelley, S. Buathong, and F. B. Dunning, J. Chem. Phys. 146, 184307 (2017).

${ }^{7}$ M. O. Vieitez, T. I. Ivanov, E. Reinhold, C. A. de Lange, and W. Ubachs, Phys. Rev. Lett. 101, 163001 (2008).

${ }^{8}$ E. Reinhold and W. Ubachs, Phys. Rev. Lett. 88, 013001 (2001).

${ }^{9}$ R. C. Shiell, E. Reinhold, F. Magnus, and W. Ubachs, Phys. Rev. Lett. 95, 213002 (2005).

${ }^{10}$ S. Mollet and F. Merkt, Phys. Rev. A 82, 032510 (2010).

${ }^{11}$ P. Allmendinger, J. A. Agner, H. Schmutz, and F. Merkt, Phys. Rev. A 88, 043433 (2013).

${ }^{12}$ P. Lancuba and S. D. Hogan, Phys. Rev. A 90, 053420 (2014).

${ }^{13}$ S. D. Hogan, EPJ Tech. Instrum. 3, 2 (2016).

${ }^{14}$ B. Brutschy and H. Haberland, J. Phys. E: Sci. Instrum. 13, 150 (1980).

${ }^{15}$ X. Ling, M. A. Durham, A. Kalamarides, R. W. Marawar, B. G. Lindsay, K. A. Smith, and F. B. Dunning, J. Chem. Phys. 93, 8669 (1990).

${ }^{16}$ C. E. Klots, J. Chem. Phys. 41, 117 (1964).

${ }^{17}$ R. A. Popple, C. D. Finch, K. A. Smith, and F. B. Dunning, J. Chem. Phys. 104, 8485 (1996).

${ }^{18}$ C. O. Reinhold, S. Yoshida, and F. B. Dunning, J. Chem. Phys. 134, 174305 (2011)

${ }^{19}$ R. Parthasarathy, L. Suess, S. B. Hill, and F. B. Dunning, J. Chem. Phys. 114, 7962 (2001).

${ }^{20}$ C. A. Gottlieb, S. Brünken, M. C. McCarthy, and P. Thaddeus, J. Chem. Phys. 126, 191101 (2007).

${ }^{21}$ C. W. Walter, B. G. Lindsay, K. A. Smith, and F. B. Dunning, Chem. Phys. Lett. 154, 409 (1989).

${ }^{22}$ R. A. Popple, C. D. Finch, and F. B. Dunning, Int. J. Mass Spectrom. Ion Processes 149-150, 37 (1995).

${ }^{23}$ V. Zhelyazkova and S. D. Hogan, Phys. Rev. A 95, 042710 (2017).

${ }^{24}$ V. Zhelyazkova and S. D. Hogan, J. Chem. Phys. 147, 244302 (2017). 